\title{
TRAMADOL VIA EPIDURAL EM CÃES SUBMETIDOS À SUBSTITUIÇÃO DO LIGAMENTO CRUZADO CRANIAL
}

\author{
THE USE OF EPIDURAL TRAMADOL IN DOGS SUBMITTED TO CRANIAL CRUCIATE \\ LIGAMENT REPLACEMENT
}

\author{
Alonso Gabriel Pereira Guedes ${ }^{1}$ Cláudio Corrêa Natalini ${ }^{2}$ Simone Dias de Lima Alves ${ }^{1}$ \\ Simone Tostes Oliveira ${ }^{1}$
}

RESUMO

Dez cães foram submetidos à substituição experimental do ligamento cruzado cranial e receberam tramadol $(1 \mathrm{mg} / \mathrm{kg})$ pela via epidural lombo-sacra como técnica analgésica trans e pós-operatória. Avaliaram-se as funções cardiovascular e respiratória, o consumo de halotano e a analgesia pós-operatória. Observou-se estabilidade hemodinâmica e respiratória, analgesia adequada durante e após a cirurgia, bem como a possibilidade de redução no consumo de anestésico inalatório. Conclui-se que o tramadol epidural é efetivo como adjuvante anestésico em cães submetidos à substituição do ligamento cruzado cranial.

Palavras-chave: anestesia, analgesia, tramadol, cães, halotano, acepromazina.

\section{SUMMARY}

Ten dogs were submitted to an experimental replacement of the cranial cruciate ligament and received epidural tramadol (1mg/kg) as a trans and post-operative analgesic technique. Cardiovascular and respiratory functions, halothane consumption and post-operative analgesia were evaluated. Cardiovascular and respiratory stability, suitable analgesia during and after the surgery as well as the possibility of reducing the inhalant analgesic consumption were observed. Epidural tramadol is effective as an adjuvant anesthetic in dogs submitted to cranial cruciate ligament replacement.
Key words: anesthesia, analgesia, tramadol, dogs, halothane, acepromazine.

O tramadol vem sendo utilizado pela via epidural em humanos (WILDER-SMITH et al., 1998). Em medicina veterinária, foi recentemente experimentado em eqüinos por NATALINI \& ROBINSON (2000), mostrando-se promissor na terapia analgésica em animais. Farmacologicamente, é um análogo opióide sintético, com ação agonista $\mu$-opióide e bloqueador da recaptação monoaminérgica (WILDER-SMITH et al., 1998). O tramadol não tem causado efeitos colaterais significativos nos sistemas cardiovascular e respiratório em humanos (LEHMANN, 1997) e em equiinos pela via epidural (NATALINI \& ROBINSON, 2000).

Neste trabalho objetivou-se determinar os efeitos cardiovasculares, respiratórios e analgésicos do tramadol administrado pela via epidural em cães submetidos à substituição experimental do ligamento cruzado cranial. Utilizaram-se acepromazina $(0,05 \mathrm{mg} / \mathrm{kg} / \mathrm{IM})$ na medicação pré-anestésica

\footnotetext{
${ }^{1}$ Médicos Veterinários, alunos do Programa de Pós-graduação em Medicina Veterinária da Universidade Federal de Santa Maria (UFSM).

${ }^{2}$ Médico Veterinário, Professor adjunto, PhD, Departamento de Clínica de Pequenos Animais, UFSM, 97105-900, Santa Maria, RS. Email: natalini@hcv.ufsm.br. Autor para correspondência.
} 
(MPA), tiopental sódico (10mg/kg/IV) na indução e halotano em oxigênio na manutenção da anestesia. Administrou-se tramadol $(1 \mathrm{mg} / \mathrm{kg})$ no espaço epidural lombo-sacro, diluído em água bidestilada a um volume final de $4,5 \mathrm{ml} / \mathrm{kg}$, após a indução anestésica.

A função cardiovascular foi adequadamente mantida, encontrando-se dentro da faixa de variação fisiológica para cães sob anestesia geral. $\mathrm{Na}$ função respiratória, houve redução significativa $(\mathrm{p}<0,05)$ na freqüência respiratória $(\mathrm{FR})$ e no $\mathrm{pH}$, enquanto as pressões parciais de oxigênio $\left(\mathrm{PaO}_{2}\right)$ e de dióxido de carbono $\left(\mathrm{PaCO}_{2}\right)$ arteriais elevaram-se $(\mathrm{p}<0,05)$ no período trans-anestésico. No período pós-operatório, não houve alteração da freqüência cardíaca $(f)$, da FR e da oximetria de pulso $\left(\mathrm{SpO}_{2}\right)$. Além disso, observaram-se ausência de vocalização e reperfusão capilar inferior a dois segundos, e os animais permaneciam calmos ou dormindo durante todo o período de avaliação.

Assim como em eqüinos (NATALINI \& ROBINSON, 2000) e em humanos (WILDERSMITH et al., 1998), o tramadol não causou interferência significativa na função hemodinâmica em cães. O fármaco mostrou-se não ser depressor da função respiratória tanto durante quanto após a cirurgia, quando administrado pela via epidural, corroborando com as informações de LEHMANN (1997) em humanos. A analgesia pós-operatória conferida pelo tramadol foi efetiva durante as quatro horas de observação, concordando com NATALINI \& ROBINSON (2000) em eqüinos.

Conclui-se que o tramadol não altera clinicamente as funções hemodinâmica e respiratória, produzindo analgesia trans-operatória adequada, que se estende por um período mínimo de quatro horas pós-operatórias em cães anestesiados com halotano e submetidos à substituição experimental do ligamento cruzado cranial.

\section{REFERÊNCIAS BIBLIOGRÁFICAS}

LEHMANN, K.A. Tramadol in acute pain. Drugs, v.53, n.2, p.25-33, 1997.

NATALINI, C.C., ROBINSON, E.P. Evaluation of the analgesic effects of epidurally administered morphine, alfentanil, butorphanol, tramadol, and U50488H in horses. Am J Vet Res, v.61, n.12, p.1579-1586, 2000.

WILDER-SMITH, C.H., WILDER-SMITH, O.H.G., FARSCHTSCHIAN, M., et al. Preoperative adjuvant epidural tramadol: the effect of different doses on postoperative analgesia and pain processing. Acta Anest Scand, v.42, n.2, p.299-305, 1998. 